\title{
Fasting circulating glicentin increases after bariatric surgery
}

Manuscript type I : original contribution

Juliette Raffort ${ }^{1,3}$, Patricia Panaïa-Ferrari ${ }^{1,2}$, Fabien Lareyre ${ }^{3,4}$, Pascale Bayer ${ }^{1,2}$, Pascal Staccini $^{2}$, Patrick Fénichel ${ }^{2,5}$, Giulia Chinetti ${ }^{1,3}$

${ }^{1}$ Clinical Chemistry Laboratory, University Hospital of Nice, France

${ }^{2}$ Université Côte d'Azur, CHU, France

${ }^{3}$ Université Côte d'Azur, CHU, CNRS, Inserm, IRCAN, France

${ }^{4}$ Department of Vascular Surgery, University Hospital of Nice, France

${ }^{5}$ Department of Endocrinology, University Hospital of Nice, France

\section{Correspondence to:}

Prof. Giulia Chinetti,

Clinical Chemistry Laboratory, University Hospital of Nice, France

30 Avenue de la Voie Romaine CS 51069

06002 Nice Cedex 1

Telephone number: +33 - 0492038163

Fax number: +33 - 0492038595

E-mail : giulia.chinetti@unice.fr

Shortened title : glicentin in bariatric surgery 
Funding: This study was supported by the Universite Côte d'Azur and the University Hospital of Nice.

Acknowledgements: Teams of the Department of Endocrinology, Bariatric and Visceral Surgery and of the Clinical Chemistry Laboratory (University Hospital of Nice) are acknowledged for their help in collecting samples and data. M. Kacimi (Mercodia ${ }^{\circledR}$ ) is acknowledged for providing glicentin Elisa kits. 


\begin{abstract}
Introduction: Bariatric surgery including the Roux-en-Y Gastric Bypass (RYGB) and the laparoscopic sleeve gastrectomy (LSG) is a well-established therapeutic option for patients with morbid or severe obesity. Metabolic modifications observed after bariatric surgery are thought to be, at least partly, linked to hormonal changes. While variation of several proglucagon-derived peptides during bariatric surgery is well documented, little is known about glicentin. The aim of this study was to investigate circulating glicentin variations after bariatric surgery.
\end{abstract}

Material and methods: Thirty patients eligible for bariatric surgery (18 RYGB and 12 LSG procedures) were prospectively included in the University Hospital of Nice. Clinical data and fasting biological parameters were recorded pre-operatively, at 3, 6 and 12 months after bariatric surgery.

Results: The median age of patients was 51 years (35-56) with $33.3 \%$ men. Fasting glicentin concentration increased progressively after bariatric surgery from 6 months and was more marked at 12 months $(14+/-3.6 \mathrm{pmol} / \mathrm{L}$ at baseline vs $19.7+/-2.7 \mathrm{pmol} / \mathrm{L}$ at 12 months for RYGB, and 12.5 +/- $1.4 \mathrm{pmol} / \mathrm{L}$ vs $16.4+/-1.8 \mathrm{pmol} / \mathrm{L}$ for LSG, respectively). Compared to pre-operative values, the fold increase of glicentin at 12 months was $2+/-0.2$ in the RYGB group and $1.6+/-0.3$ in the LSG group. Glicentin variation after surgery did not correlate with anthropometric, glycemic or lipid parameter modifications.

Conclusion: Fasting glicentin level increases after bariatric surgery suggesting the potential interest of this peptide as player and/or marker of physiological changes after bariatric surgery. 
Abbreviations:

ALT: aspartate aminotransferase

AST: alanine aminotransferase

BMI: Body Mass Index

GGT: gamma glutamyltransferase

GLP-1: Glucagon-Like Peptide-1

GLP-2: Glucagon-Like Peptide-2

HDL: High Density Lipoprotein

HOMA-IR: Homeostasis model assessment for insulin resistance

LDL: Low Density Lipoprotein

LSG: Laparoscopic Sleeve Gastrectomy

RYGB: Roux-en-Y Gastric Bypass

Key words: glicentin, obesity, bariatric surgery, Roux-en-Y Gastric Bypass, Laparoscopic Sleeve Gastrectomy 


\section{Introduction}

Obesity is a leading cause of morbidity and mortality and its increasing worldwide prevalence represents a major public health concern [1]. Bariatric surgery has proven its efficiency to induce weight loss and improve obesity related comorbidities and has become an attractive therapeutic option [2, 3]. Several guidelines describing eligibility for bariatric surgery have been elaborated and this treatment is currently proposed to patients with morbid obesity (BMI $\left.>40 \mathrm{~kg} / \mathrm{m}^{2}\right)$ or with severe obesity $\left(\mathrm{BMI}>35 \mathrm{~kg} / \mathrm{m}^{2}\right)$ associated with comorbidities [4].

The Roux-en-Y Gastric Bypass (RYGB) is one of the most commonly used procedures [5]. This technique consists in reducing the size of the stomach to a small pouch and connecting it to the small intestine, shunting the duodenum and a portion of the jejunum. This induces restrictive and malabsorptive effects leading to weight loss [6]. Restrictive surgery such as the Laparoscopic Sleeve Gastrectomy (LSG) which consists in a longitudinal resection of the stomach can also be performed [6]. Both procedures have proven their efficiency for longlasting weight loss and improvement of metabolic parameters such as transient amelioration of diabetes, glucose tolerance or lipid profiles $[2,3,6]$. Among the mechanisms involved in metabolic improvement, the modification of gut hormone secretion including proglucagonderived hormones appears to play a key role [7-10].

The proglucagon gene is mainly expressed in the pancreatic alpha-cells as well as in the intestinal L-cells $[11,12]$ and leads to the synthesis of the proglucagon peptide. In the pancreas, this precursor is cleaved in several peptides including the glucagon [11]. In the entero-endocrine L-cells, post-translational processing of proglucagon liberates other peptides such as Glucagon-Like Peptide-1 (GLP-1), Glucagon-Like Peptide-2 (GLP-2) [11], oxyntomodulin or glicentin. While the role of GLP-1, GLP-2, oxyntomodulin or glucagon is well known and their variation well documented in obese patients who underwent bariatric 
surgery $[7-10,13,14]$, little is known about glicentin. This could be attributed, at least partly, to the lack of commercialized detection methods until recently. Studies on animal models have reported the effect of glicentin on gastric acid secretion [15], intestinal motility and trophicity [16-18] as well as an inhibitory effect on insulin secretion [19]. Experimental studies suggest a role of glicentin in intestinal physiology and metabolism regulation [15-19]. Nevertheless, circulating glicentin levels during adult obesity are poorly known and its variation after bariatric surgery has never been described so far. The aim of our study was to investigate glicentin variation in obese patients who underwent bariatric surgery.

\section{Material and methods}

\section{Population}

Obese patients eligible for bariatric surgery were prospectively included at the University Hospital of Nice between January 2014 and April 2016. Eligibility for bariatric surgery was defined as morbid obesity (BMI $\left.>40 \mathrm{~kg} / \mathrm{m}^{2}\right)$ or severe obesity $\left(\mathrm{BMI}>35 \mathrm{~kg} / \mathrm{m}^{2}\right)$ with associated comorbidities (type 2 diabetes, arterial hypertension or sleep apnea). The decision for surgical treatment was approved by a multidisciplinary staff. Inclusion criteria were patients aged between 18 and 65 years old with a stable weight (+/- 5kg over a 3 month-period). Exclusion criteria were defined as the presence of type 1 diabetes or insulin treatment for type 2 diabetic subjects. Written informed consent was obtained from all individual participants included in the study. All procedures performed in this study were in accordance with the ethical standards of the local ethics committee of the University Hospital of Nice and with the 1964 Helsinki Declaration and its later amendments or comparable ethical standards.

Obese patients eligible for bariatric surgery had a clinical evaluation and biological measurements at baseline, the day before the surgery. Technical procedures performed for 
bariatric surgery were LSG or RYGB according to the decision made by the multidisciplinary staff. After the surgery, patients had a follow-up visit at 3, 6, and 12 months. BMI was calculated according to the formula: weight $(\mathrm{kg}) /$ height $^{2}(\mathrm{~m})$. Fat mass was evaluated by dual energy x-ray absorptiometry (DEXA) or by impedancemetry. Homeostasis model assessment for insulin resistance (HOMA-IR) was calculated according to the formula: glycemia $(\mathrm{mmol} / \mathrm{L}) \mathrm{x}$ insulinemia $(\mathrm{mU} / \mathrm{L}) / 22.5$.

\section{Biochemical analysis}

All biological parameters were measured after a peripheral vein puncture on fasting patients. Blood samples for determination of insulin, C-peptide, glycemia, total cholesterol, HDLcholesterol, LDL-cholesterol, triglyceride, leptin and glicentin were collected in vacutainer tubes with serum separator and clot activator. For glycemia, aspartate aminotransferase (AST), alanine aminotransferase (ALT), gamma glutamyl-transferase (GGT), alkaline phosphatase and bilirubin measurement, blood was collected in tubes coated with lithium heparin. For HbA1c measure blood was collected in K3EDTA-containing tubes. Samples were centrifuged at room temperature for 15 minutes at $3000 \mathrm{rpm}$ and sera were stored at $80^{\circ}$. All the analyses were performed in the Laboratory of Clinical Biochemistry at the University Hospital of Nice.

Glycemia, total cholesterol, HDL-cholesterol triglyceride, AST, ALT, GGT, alkaline phosphatase and bilirubin concentrations were determined by spectrophotometry (Cobas 8000 , module 700 or 500 , Roche $\left.{ }^{\circledR}\right)$. Insulin and C-peptide were measured with a sandwich immunoassay using direct chemiluminescent technology (Centaur Xp, Siemens ${ }^{\circledR}$ ). HbA1c was measured by high-performance liquid chromatography method (Variant II, Biorad®). Leptin was determined by a sandwich Elisa method (Teco®). For all assays, quality controls 
fell within predefined limits.

Glicentin was measured by Elisa technique (Mercodia ${ }^{\circledR}$ ). The glicentin assay uses an antibody against the glucagon sequence and the other recognizing the glicentin related polypeptide sequence. The assay was performed according to the manufacturer's protocol. All values were above the detection limit ( $3 \mathrm{pmol} / \mathrm{L})$. All samples were analyzed in duplicates and the precision coefficients (within-assay and inter-assay) were determined and were consistent with the recommendations of the manufacturer.

\section{Statistical analysis}

Categorical data were expressed as the number of patients and percentage, whereas continuous variables were expressed as the median values with interquartile ranges. For the follow-up of patients, data were expressed as means +/- standard error means. Data were analyzed using a repeated-measures ANOVA followed by a Bonferroni post-test. Correlations were determined by non-parametric univariate linear regression (Spearman's rank test). A P value $<0.05$ was considered as significant. Statistical analyses were performed using GraphPad Prism ${ }^{\circledR}$ software (version 7.00, La Jolla California USA).

\section{Results}

Baseline characteristics of the population

Thirty-nine patients eligible for bariatric surgery $\left(\mathrm{BMI}>40 \mathrm{~kg} / \mathrm{m}^{2}\right.$ or $\mathrm{BMI}>35 \mathrm{~kg} / \mathrm{m}^{2}$ with obesity-related comorbidities) were included in the study at baseline (Figure 1). Nine patients were lost during the follow-up period. In total, 30 patients were followed at all time points 
(pre-operative baseline and at 3, 6 and 12 months after the intervention) and were finally included for the data analyses. Among them, 18 (60\%) underwent a RYGB procedure and 12 (40\%) had a LSG.

The median age of patients was 51 years $(35-56)$ and $33.3 \%$ of them were men (Table 1$)$. The median BMI was $42.7 \mathrm{~kg} / \mathrm{m}^{2}(39.7-44.5)$. The median of abdominal circumference and fat mass proportion were $130 \mathrm{~cm}(125.5-137.3)$ and $51.5 \%$ (44.5-55.3), respectively. Eight patients $(26.7 \%)$ had arterial hypertension, 5 (16.7\%) had type 2 diabetes, 10 (33.3\%) dyslipidemia, 4 (13.3\%) hyperuricemia and 12 (40\%) suffered from sleep apnea. Clinical and biological characteristics were comparable between patients who underwent RYGB and those who underwent LSG.

\section{Variation of glicentin after bariatric surgery}

Mean pre-operative fasting glicentin concentrations were similar between patients who underwent RYGB procedure compared to those who had LSG (14 +/- 3.6 pmol/L vs $12.5+/$ 1.4, respectively) (Figure 2a). At 3 months, blood glicentin concentration did not significantly vary compared to baseline. Glicentin concentration tended to increase at 6 months for both groups. This effect was more pronounced at 12 months and reached statistical significance in the RYGB group ( $\mathrm{p}=0.04)$. To better assess glicentin fold increase after bariatric surgery, post-operative values were reported to pre-operative values for each patient (Figure 2b). In both group, glicentin increased post-operatively from 6 months and the effect was accentuated and statistically significant at 12 months for both group, with a fold increase of $2+/-0.2$ in the RYGB group and 1.6 +/- 0.3 in the LSG group. At all post-operative time points, glicentin concentrations and fold increase tended to be higher in the RYGB group even if the difference between the 2 techniques did not reach statistical significance. 
Experimental studies have reported an involvement of glicentin in glucose homeostasis through an action on insulin secretion [19]. To investigate the potential link between glicentin and glucose homeostasis in the context of bariatric surgery, the ratio between glicentin concentrations and glycemia, insulinemia and C-peptide levels (Figure 3) were calculated. Ratio of glicentin/ glycemia tended to increase over time in patients who had RYGB and LSG (Figure 3a). This increase was statistically significant at 6 and 12 months for the RYGB group. Ratio of glicentin/ insulinemia progressively increased over time in both group and was statistically significant from 3 months in the RYGB group $(1.5+/-0.4$ vs $0.5+/-0.1$ at baseline, $\mathrm{p}=0.01)$ and from 6 months in the LSG group $(1.9+/-0.3$ vs $0.6+/-0.1$ at baseline, $\mathrm{p}=0.02$ ) (Figure 3b). Ratio of glicentin/ C-peptide varied in the same way as glicentin/ insulinemia ratio, with a progressive increase over time which was statistically significant from 6 months in both group $(3.7+/-0.6$ vs $1.2+/-0.1$ at baseline, $\mathrm{p}<0.0001$ in the RYGB group and $3.3+/-0.6$ vs $1.7+/-0.3$ at baseline, $p=0.04$ in the LSG group) (Figure $3 \mathrm{c}$ ). Ratio of glicentin/ insulinemia and glicentin/ C-peptide tended to be higher in the RYGB group than in the LSG group at all post-operative time points, but the difference between the 2 groups did not reach statistical significance.

\section{Link between glicentin variation and other metabolic parameters}

To investigate whether variations of glicentin levels observed after bariatric surgery are associated with modifications of other metabolic parameters, potential correlation were searched. A significant and progressive improvement of anthropometric parameters including a decrease of BMI, fat mass and abdominal circumference was observed from 3 months for both surgical procedures (Figure S1). At 12 months, weight loss was comparable between the two techniques (28.8 \% +/- 2.6 for RYGB vs $32.1 \%+/-1.9$ for LSG). For both procedures, 
an improvement of glycemic parameters was observed from 3 months and was maintained over the 12 month-follow-up period (Figure S2). This included lower fasting glycemia, lower HbAlc levels and a decrease of insulin resistance revealed by a reduction of insulinemia, Cpeptide levels and HOMA-IR index. At last, both techniques showed a beneficial effect on lipid parameters with a decrease of triglyceride levels, accompanied by an increase of HDLcholesterol concentration (Figure S3). A significant decrease of total cholesterol and LDLcholesterol was observed in the RYGB group but not in the LSG group.

Since metabolic modifications as well as glicentin variation were more marked at 12 months after bariatric surgery, we explored their potential correlations at this time point (Table 2). Glicentin variation did not correlate with glycemic and lipid variation, as revealed by Spearman coefficient correlations close to zero. For anthropometric parameters, Spearman coefficient correlations were slightly higher $(0.22 ; 0.22$ and 0.37 for BMI, fat mass and abdominal circumference variation, respectively) but dot plots showed no correlation between glicentin and anthropometric parameter variations.

\section{Discussion}

In the present study, we investigated the impact of bariatric surgery on blood circulating glicentin levels. During the one-year post-operative follow-up period, fasting glicentin levels progressively increased in patients who underwent RYGB or LSG. The effect of bariatric surgery on glicentin variation tended to be more pronounced in patients who underwent RYGB, even if the difference between the two techniques did not reach statistical significance.

So far, glicentin variation in the context of obesity has been poorly investigated. Our personal work in another cohort of 52 lean subjects $\left(\mathrm{BMI}<25 \mathrm{~kg} / \mathrm{m}^{2}\right)$ and 39 patients with severe or 
morbid obesity (BMI $\left.>35 \mathrm{~kg} / \mathrm{m}^{2}\right)$ revealed that obesity was significantly associated with lower circulating glicentin (submitted data). Furthermore, a previously published study reports lower glicentin levels in adolescents with obesity and impaired glucose tolerance compared to those with obesity and normal glucose tolerance [20], suggesting that glicentin alteration during obesity could be related to metabolic disorders.

Interestingly, our study reveals that bariatric surgery may, at least partially, restore fasting circulating glicentin concentration. To the best of our knowledge, this is the first study reporting the variations of glicentin levels after bariatric surgery. A published study analyzed the glicentin levels in the context of digestive surgery [21]. The investigators observed a higher response of glicentin levels after glucose load in patients who underwent gastrectomy as compared to control group, whereas no significant changes occurred in a patient with massive short bowel syndrome. These results suggest that surgical procedures may affect differently glicentin secretion. This hypothesis is corroborated by the fact that variation of glicentin tended to be more marked after RYGB compared to LSG.

To better understanding the physiology of glicentin production, some authors investigated the intestinal distribution pattern of pro-glucagon derived hormones by using several animal models (mouse, rat and pig). They showed that tissue concentration of oxyntomodulin/ glicentin were higher in distal ileum, caecum, and proximal colon compared to duodenum and proximal jejunum in the 3 species [22]. These results corroborate the fact that enteroendocrine L cells, responsible for the intestinal production of proglucagon-derived hormones, are present in duodenum to rectum but are rare before terminal ileum [23]. Hence, we can hypothesize that bariatric surgery with LSG and RYGB techniques saves the main intestinal regions involved in glicentin production and that modifications induced by surgery may act as stimulating factors for its secretion. 
Experimental models have demonstrated the role of glicentin in intestinal trophicity and motility [16-18, 24, 25]. As bariatric surgery modifies intestinal physiology and impacts on various processes including motility, nutrient digestion and absorption [26], it is possible that post-surgery intestinal remodeling could act as a stimulating factor for glicentin secretion.

Food intake and ingestion of nutrients including glucose, lipids or amino-acids have been identified to act as stimulating factors for glicentin secretion [21, 27-29]. In that context, two main hypotheses previously described by other authors can be proposed and transposed to explain glicentin increase after bariatric surgery: the hindgut and the foregut hypothesis [30, 31]. Both LSG and RYGB procedures lead to a drastic reduction of the gastric size $[6,26]$. This contributes to modify the intestinal transit and may induce a rapid stimulation of the distal ileum. The hindgut hypothesis suggests that the rapid stimulation of the distal ileum by nutrients could act as a stimulating factor and this could partly explain the increase of circulating glicentin observed post-operatively in patients who underwent LSG and RYGB. In addition to reduce the gastric food reservoir, the RYGB procedure also associates malabsorptive effects by excluding the duodenum and the proximal jejunum [6]. In the foregut hypothesis, we can suggest that the exclusion of the upper gut could indirectly contribute to stimulate glicentin secretion. This could partly explain why the effect on circulating glicentin concentration tended to be more pronounced in patients who underwent RYGB procedure compared to LSG.

In addition to major intestinal changes, bariatric surgery is associated with various metabolic and hormonal modifications [2, 3, 6, 32]. Both RYGB and LSG induced a drastic weight loss during the follow-up period, concomitantly with an improvement of glycemic parameters and insulin resistance, a decrease of triglyceride and an increase of HDL-cholesterol. A reduction of total and LDL-cholesterol was observed in the RYGB group but not in the LSG group, which is in agreement with previous studies [33, 34]. As glycemic parameters including 
glycemia, insulinemia and C-peptide decreased post-operatively and glicentin progressively increased, it is not surprising that ratios of glicentin reported to glycemic parameters increased over time. Even if we did not observe a correlation at 12 months between glicentin variation and anthropometric, glycemic or lipid variation, it remains possible that metabolic modifications induced by surgery may impact on glicentin secretion. Studies in animal models revealed the involvement of glicentin in glucose homeostasis through a stimulatory action on insulin secretion and an inhibitory effect on glucagon secretion $[19,35,36]$. We can hypothesize that in return, insulin may have a negative feed-back on glicentin secretion and decreased insulin levels after bariatric surgery could have a positive action on glicentin secretion.

During the past few years, several studies have highlighted major post-operative hormonal changes induced by bariatric surgery $[7,13,14]$. Contrarily to glicentin, the variation of the other members of the proglucagon-derived hormones, including GLP-1, GLP-2 and oxyntomodulin, has been well described and even if some results were heterogeneous among different studies, some data obtained pinpoint an increase of these hormones after bariatric surgery $[7,13,37]$. The production of these four proglucagon-derived peptides results from the post-translational processing of the proglucagon by the proconvertases 1 and 3 in the intestinal L-cells and their secretion is stimulated by food intake [11, 38, 39]. Further experimental and clinical studies would be of interest to better understand the link between each member of the proglucagon family and confirm if these molecules are co-secreted and vary similarly after bariatric surgery.

Interestingly, the modification of the secretion of some proglucagon-derived hormones such as GLP-1 has been identified to play key role in improvement of glucose homeostasis after bariatric surgery [14]. Hence, glicentin increase after bariatric surgery could potentially play a role in the modifications of glucose homeostasis observed after bariatric surgery. Further 
clinical and experimental studies would be of interest to determine factors involved in glicentin increase after bariatric surgery and to better understand its clinical significance.

It would be worth to extend this work on larger cohorts to improve statistical power to better assess the impact of each surgical technique (RYGB or LSG) on glicentin level. In addition, to better evaluate the link between glicentin and glucose homeostasis, it would be interesting to study modifications of glicentin levels after glucose ingestion during the follow-up period post-surgery.

\section{Conclusion}

In this study, we investigated glicentin variation in obese patients who underwent bariatric surgery. A progressive and significant increase of fasting glicentin levels was observed during the one-year follow-up period in patients who underwent RYGB or LSG. The effect of surgery on glicentin tended to be more pronounced in the RYGB group compared to LSG, but the difference between the two techniques was not statistically significant. After the surgery, an improvement of anthropometric, glycemic and lipid profiles was observed but their variation did not correlate with glicentin variation. Glicentin has been described to be involved in intestinal physiology as well as glucose homeostasis. Both intestinal remodeling and metabolic changes could be potential factors impacting on glicentin secretion post-surgery. Even if further studies are required to establish its clinical significance, glicentin could represent an interesting player and/or marker of metabolic and physiological changes that occur after bariatric surgery.

Conflict of interest disclosure statement: All the authors declare that they have no conflict 
of interest.

Author contributions: All authors contributed to design, conduct, collect, analyze and interpret the data. All authors contributed to the intellectual content of this article and approved the final version.

Statement of informed consent: Informed consent was obtained from all individual participants included in the study.

Statement of Human and Animal Rights: All procedures performed in studies involving human participants were done in accordance with the ethical standards of the institutional and/or national research committee and with the 1964 Helsinki declaration and its later amendments or comparable ethical standards. 


\section{References:}

1. http://www.who.int/gho/ncd/risk_factors/overweight/en/

2. Sjostrom L, Lindroos AK, Peltonen M, Torgerson J, Bouchard C, Carlsson B, et al. Lifestyle, diabetes, and cardiovascular risk factors 10 years after bariatric surgery. N Engl J Med. 2004;351(26):2683-93.

3. Buchwald H, Estok R, Fahrbach K, Banel D, Jensen MD, Pories WJ, et al. Weight and type 2 diabetes after bariatric surgery: systematic review and meta-analysis. Am J Med. 2009;122(3):248-56.

4. Jensen MD, Ryan DH, Apovian CM, Ard JD, Comuzzie AG, Donato KA, et al. 2013 AHA/ACC/TOS guideline for the management of overweight and obesity in adults: a report of the American College of Cardiology/American Heart Association Task Force on Practice Guidelines and The Obesity Society. Circulation. 2014;129(25 Suppl 2):S102-38.

5. Buchwald H, Oien DM. Metabolic/bariatric surgery Worldwide 2008. Obes Surg. 2009;19(12):1605-11.

6. Piche ME, Auclair A, Harvey J, Marceau S, Poirier P. How to choose and use bariatric surgery in 2015. Can J Cardiol. 2015;31(2):153-66.

7. Holst JJ. Enteroendocrine secretion of gut hormones in diabetes, obesity and after bariatric surgery. Curr Opin Pharmacol. 2013;13(6):983-8.

8. Papamargaritis D, Miras AD, le Roux CW. Influence of diabetes surgery on gut hormones and incretins. Nutr Hosp. 2013;28 Suppl 2:95-103.

9. Ionut V, Burch M, Youdim A, Bergman RN. Gastrointestinal hormones and bariatric surgery-induced weight loss. Obesity (Silver Spring). 2013;21(6):1093-103.

10. Dirksen C, Jorgensen NB, Bojsen-Moller KN, Jacobsen SH, Hansen DL, Worm D, et al. Mechanisms of improved glycaemic control after Roux-en-Y gastric bypass. Diabetologia. 2012;55(7):1890-901. 
11. Baggio LL, Drucker DJ. Biology of incretins: GLP-1 and GIP. Gastroenterology. 2007;132(6):2131-57.

12. Holst JJ. Enteroglucagon. Annu Rev Physiol. 1997;59:257-71.

13. Hage MP, Safadi B, Salti I, Nasrallah M. Role of Gut-Related Peptides and Other Hormones in the Amelioration of Type 2 Diabetes after Roux-en-Y Gastric Bypass Surgery. ISRN Endocrinol. 2012;2012:504756.

14. Sala PC, Torrinhas RS, Giannella-Neto D, Waitzberg DL. Relationship between gut hormones and glucose homeostasis after bariatric surgery. Diabetol Metab Syndr. 2014;6(1):87.

15. Kirkegaard P, Moody AJ, Holst JJ, Loud FB, Olsen PS, Christiansen J. Glicentin inhibits gastric acid secretion in the rat. Nature. 1982;297(5862):156-7.

16. Myojo S, Tsujikawa T, Sasaki M, Fujiyama Y, Bamba T. Trophic effects of glicentin on rat small-intestinal mucosa in vivo and in vitro. J Gastroenterol. 1997;32(3):300-5.

17. Pellissier S, Sasaki K, Le-Nguyen D, Bataille D, Jarrousse C. Oxyntomodulin and glicentin are potent inhibitors of the fed motility pattern in small intestine. Neurogastroenterol Motil. 2004;16(4):455-63.

18. Tomita R, Igarashi S, Tanjoh K, Fujisaki S. Role of recombinant human glicentin in the normal human jejunum: an in vitro study. Hepatogastroenterology. 2005;52(65):1459-62. 19. Ohneda A, Ohneda K, Nagasaki T, Sasaki K. Insulinotropic action of human glicentin in dogs. Metabolism. 1995;44(1):47-51.

20. Manell H, Staaf J, Manukyan L, Kristinsson H, Cen J, Stenlid R, et al. Altered Plasma Levels of Glucagon, GLP-1 and Glicentin During OGTT in Adolescents With Obesity and Type 2 Diabetes. J Clin Endocrinol Metab. 2016;101(3):1181-9.

21. Naito H, Ohneda A, Kojima R, Sato T, Sasaki K, Funayama Y, et al. Plasma glicentin in diabetic and gastrectomized patients. Regul Pept. 1999;79(1):55-61. 
22. Wewer Albrechtsen NJ, Kuhre RE, Torang S, Holst JJ. The intestinal distribution pattern of appetite- and glucose regulatory peptides in mice, rats and pigs. BMC Res Notes. 2016;9:60.

23. Gunawardene AR, Corfe BM, Staton CA. Classification and functions of enteroendocrine cells of the lower gastrointestinal tract. Int J Exp Pathol. 2011;92(4):219-31. 24. Rodier G, Magous R, Mochizuki T, Le Nguyen D, Martinez J, Bali JP, et al. Glicentin and oxyntomodulin modulate both the phosphoinositide and cyclic adenosine monophosphate signaling pathways in gastric myocytes. Endocrinology. 1999;140(1):22-8.

25. Ayachi SE, Borie F, Magous R, Sasaki K, le Nguyen D, Bali JP, et al. Contraction induced by glicentin on smooth muscle cells from the human colon is abolished by exendin (9-39). Neurogastroenterol Motil. 2005;17(2):302-9.

26. Quercia I, Dutia R, Kotler DP, Belsley S, Laferrere B. Gastrointestinal changes after bariatric surgery. Diabetes Metab. 2014;40(2):87-94.

27. Ohneda A, Takahashi H, Maruyama Y. Response of plasma glicentin to fat ingestion in piglets. Diabetes Res Clin Pract. 1987;3(2):103-9.

28. Ohneda A, Kobayashi T, Nihei J, Takahashi H. Effect of intraluminal administration of amino acids upon plasma glicentin. Diabetes Res Clin Pract. 1988;5(4):265-70.

29. Shimizu T, Tadokoro R, Kaneko N, Suzuki M, Tanaka K, Shinohara K, et al. Effects of extremely early enteral feeding on plasma glicentin levels in very-low-birthweight infants. J Paediatr Child Health. 2006;42(10):636-9.

30. Cummings DE, Overduin J, Foster-Schubert KE. Gastric bypass for obesity: mechanisms of weight loss and diabetes resolution. J Clin Endocrinol Metab. 2004;89(6):2608-15.

31. Rubino F, Forgione A, Cummings DE, Vix M, Gnuli D, Mingrone G, et al. The mechanism of diabetes control after gastrointestinal bypass surgery reveals a role of the 
proximal small intestine in the pathophysiology of type 2 diabetes. Ann Surg. 2006;244(5):741-9.

32. Rhee NA, Wahlgren CD, Pedersen J, Mortensen B, Langholz E, Wandall EP, et al. Effect of Roux-en-Y gastric bypass on the distribution and hormone expression of smallintestinal enteroendocrine cells in obese patients with type 2 diabetes. Diabetologia. 2015;58(10):2254-8.

33. Zhang F, Strain GW, Lei W, Dakin GF, Gagner M, Pomp A. Changes in lipid profiles in morbidly obese patients after laparoscopic sleeve gastrectomy (LSG). Obes Surg. 2011;21(3):305-9.

34. Oliveira Cda S, Beserra BT, Cunha RS, Brito AG, de Miranda RC, Zeni LA, et al. Impact of Roux-en-Y gastric bypass on lipid and inflammatory profiles. Rev Col Bras Cir. 2015;42(5):305-10.

35. Ohneda A, Ohneda M. Effect of glicentin-related peptides upon the secretion of insulin and glucagon in the canine pancreas. Tohoku J Exp Med. 1988;155(2):197-204.

36. Ohneda A, Kobayashi T, Nihei J. Effect of glicentin-related peptides on glucagon secretion in anaesthetized dogs. Diabetologia. 1986;29(6):397-401.

37. Yousseif A, Emmanuel J, Karra E, Millet Q, Elkalaawy M, Jenkinson AD, et al. Differential effects of laparoscopic sleeve gastrectomy and laparoscopic gastric bypass on appetite, circulating acyl-ghrelin, peptide YY3-36 and active GLP-1 levels in non-diabetic humans. Obes Surg. 2014;24(2):241-52.

38. Bataille D. Pro-protein convertases in intermediary metabolism: islet hormones, brain/gut hormones and integrated physiology. J Mol Med (Berl). 2007;85(7):673-84.

39. Bataille D, Dalle S. The forgotten members of the glucagon family. Diabetes Res Clin Pract. 2014;106(1):1-10. 
Tables: 2

Figure: 3

\section{Figure legends:}

Figure 1: Flow chart of the studied population

Figure 2: (a) Fasting glicentin levels before and at 3, 6 and 12 months after bariatric surgery, expressed as mean (pmol/L) $\pm \mathrm{SEM}$, (b) Ratio of glicentin post-operative value (post-op)/ glicentin pre-operative value (pre-op). For statistical analysis, the ratio at baseline has been set to 1 .

$* \mathrm{p}<0.05$ vs pre-operative value

$\mathrm{p}<0.05$ vs 3 months value

Pre-op: pre-operative

Post-op: post-operative

$3 \mathrm{M}: 3$ months after surgery

6 M: 6 months after surgery

$12 \mathrm{M}: 12$ months after surgery

Figure 3: Glicentin concentrations reported to glycemic parameters before and at 3, 6 and 12 months after bariatric surgery, expressed as means \pm SEM. (a) Ratio of glicentin concentration (pmol/L) reported to glycemia (mmol/L), (b) Ratio of glicentin concentration (pmol/L) 
reported to insulinemia (mU/L), (c) Ratio of glicentin concentration (pmol/L) reported to Cpeptide (pmol/L) x 100 .

$* \mathrm{p}<0.05$ vs pre-operative value

$\mathrm{p}<0.05$ vs 3 months value

Pre-op: pre-operative

$3 \mathrm{M}$ : 3 months after surgery

$6 \mathrm{M}: 6$ months after surgery

$12 \mathrm{M}: 12$ months after surgery

Figure legends supplemental data:

Figure S1: Anthropometric measures before and at 3,6 and 12 months after bariatric surgery, expressed as means \pm SEM. (a) Body mass index $\left(\mathrm{kg} / \mathrm{m}^{2}\right)$, (b) Weight loss (\%), (c) Fat mass (\%), (d) Abdominal circumference (cm).

a: $\mathrm{p}<0.05$ vs pre-operative value

b: $\mathrm{p}<0.05$ vs 3 months value

c: $\mathrm{p}<0.05$ vs 6 months value

Pre-op: pre-operative

$3 \mathrm{M}: 3$ months after surgery

$6 \mathrm{M}: 6$ months after surgery 
$12 \mathrm{M}: 12$ months after surgery

Figure S2: Glycemic parameters before and at 3, 6 and 12 months after bariatric surgery, expressed as means \pm SEM. (a) Glycemia (mmol/L), (b) HbAlc (\%), (c) Insulinemia (mU/l), (d) C-peptide (pmol/L), (E) HOMA-IR.

a: $\mathrm{p}<0.05$ vs pre-operative value

Pre-op: pre-operative

3 M: 3 months after surgery

6 M: 6 months after surgery

$12 \mathrm{M}: 12$ months after surgery

Figure S3: Lipid parameters before and at 3, 6 and 12 months after bariatric surgery, expressed as means \pm SEM. (a) Triglyceride (g/L), (b) Total cholesterol (g/L), (c) LDLcholesterol (g/L), (d) HDL-cholesterol (g/L).

a: $\mathrm{p}<0.05$ vs pre-operative value

b: $\mathrm{p}<0.05$ vs 3 months value

Pre-op: pre-operative

$3 \mathrm{M}: 3$ months after surgery

$6 \mathrm{M}$ : 6 months after surgery

$12 \mathrm{M}$ : 12 months after surgery 


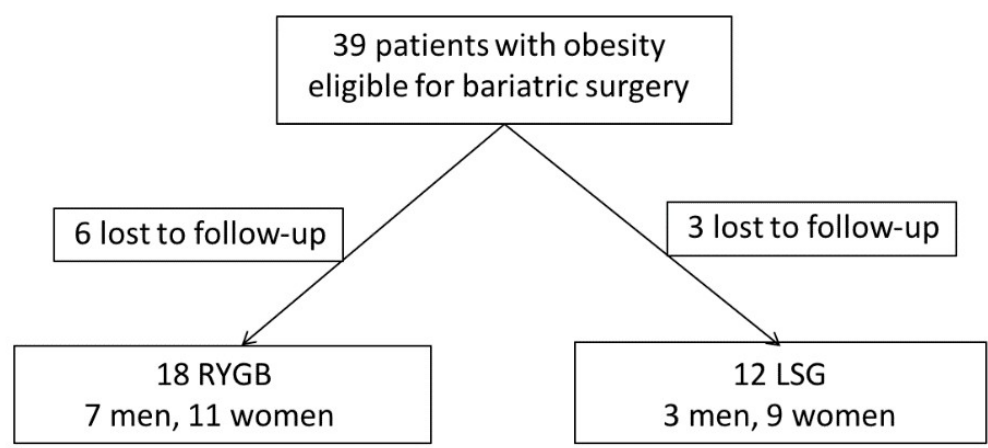

Figure 1 
a

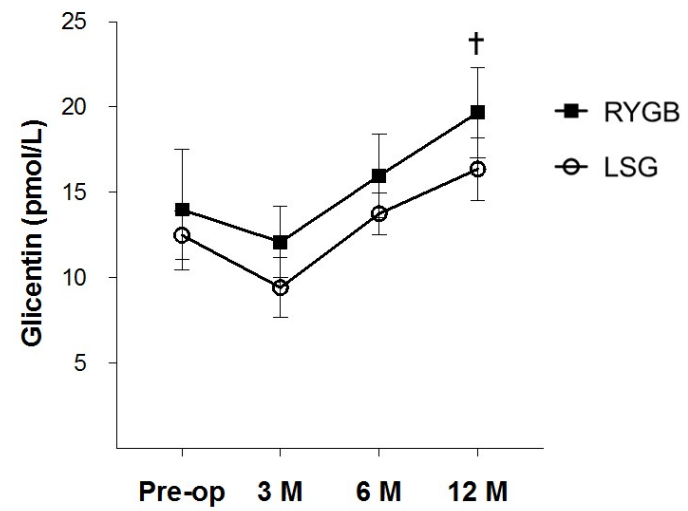

b

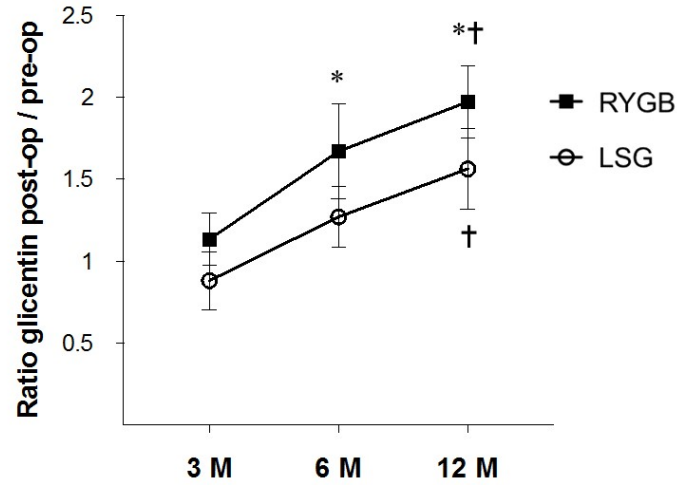

\begin{tabular}{|c|c|c|c|c|}
\hline & Pre-op & $\mathbf{3} \mathbf{M}$ & $\mathbf{6} \mathbf{M}$ & $\mathbf{1 2} \mathbf{M}$ \\
\hline $\begin{array}{c}\text { RYGB } \\
(\mathrm{n}=18)\end{array}$ & $14+/-3.6$ & $12.1+/-2.1$ & $16+/-2.5$ & $19.7+/-2.7$ \\
\hline $\begin{array}{c}\text { LSG } \\
(\mathrm{n}=12)\end{array}$ & $12.5+/-1.4$ & $9.4+/-1.7$ & $13.8+/-1.2$ & $16.4+/-1.8$ \\
\hline
\end{tabular}

\begin{tabular}{|c|c|c|c|}
\hline & $\mathbf{3} \mathbf{M}$ & $\mathbf{6} \mathbf{M}$ & $\mathbf{1 2} \mathbf{M}$ \\
\hline $\begin{array}{c}\text { RYGB } \\
(n=18)\end{array}$ & $1.1+/-0.2$ & $1.7+/-0.3$ & $2+/-0.2$ \\
\hline $\begin{array}{c}\text { LSG } \\
(n=12)\end{array}$ & $0.9+/-0.2$ & $1.3+/-0.2$ & $1.6+/-0.2$ \\
\hline
\end{tabular}

Figure 2 
a

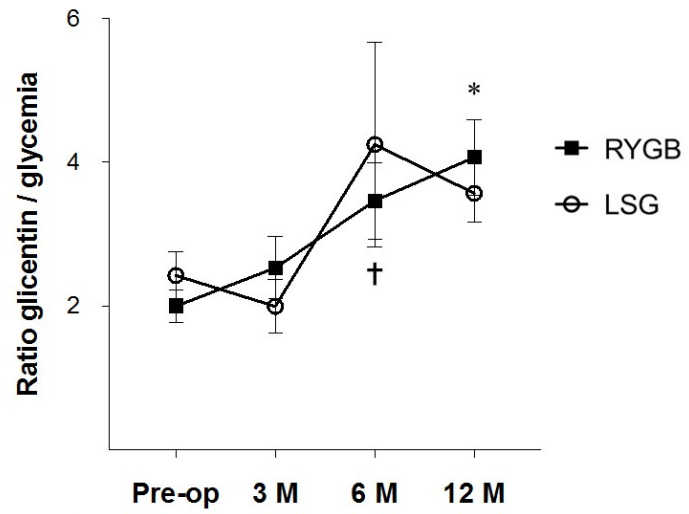

b

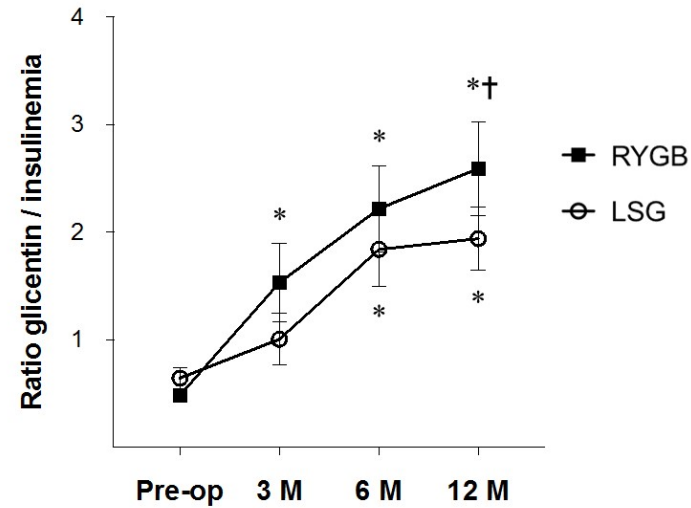

c

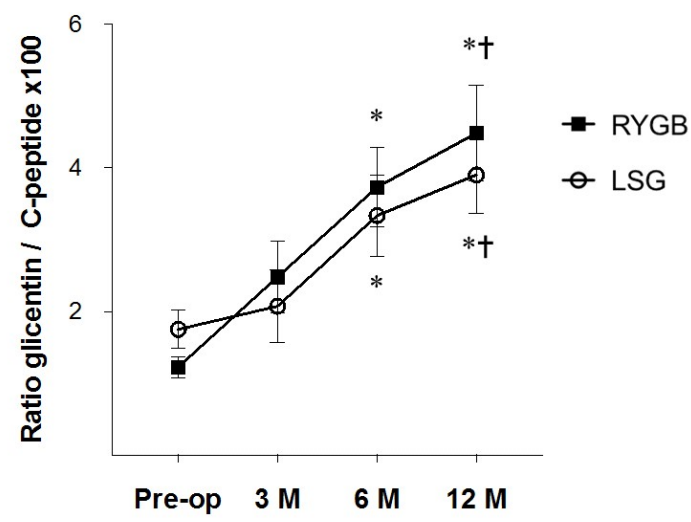

\begin{tabular}{|c|c|c|c|c|}
\hline & Pre-op & $\mathbf{3} \mathbf{~ M}$ & $\mathbf{6} \mathbf{~ M}$ & $\mathbf{1 2} \mathbf{~ M}$ \\
\hline $\begin{array}{c}\text { RYGB } \\
(\mathrm{n}=18)\end{array}$ & $2+/-0.2$ & $2.5+/-0.4$ & $3.5+/-0.5$ & $4.1+/-0.5$ \\
\hline $\begin{array}{c}\text { LSG } \\
(\mathrm{n}=12)\end{array}$ & $2.4+/-0.3$ & $2+/-0.4$ & $4.2+/-1.4$ & $3.6+/-0.4$ \\
\hline
\end{tabular}

\begin{tabular}{|c|c|c|c|c|}
\hline & Pre-op & $\mathbf{3} \mathbf{~ M}$ & $\mathbf{6} \mathbf{M}$ & $\mathbf{1 2} \mathbf{~ M}$ \\
\hline $\begin{array}{c}\text { RYGB } \\
(\mathrm{n}=18)\end{array}$ & $0.5+/-0.1$ & $1.5+/-0.4$ & $2.2+/-0.4$ & $2.6+/-0.4$ \\
\hline $\begin{array}{c}\text { LSG } \\
(\mathrm{n}=12)\end{array}$ & $0.6+/-0.1$ & $1+/-0.2$ & $1.8+/-0.3$ & $1.9+/-0.3$ \\
\hline
\end{tabular}

\begin{tabular}{|c|c|c|c|c|}
\hline & Pre-op & $\mathbf{3} \mathbf{M}$ & $\mathbf{6} \mathbf{M}$ & $\mathbf{1 2} \mathbf{M}$ \\
\hline $\begin{array}{c}\text { RYGB } \\
(n=18)\end{array}$ & $1.2+/-0.1$ & $2.5+/-0.5$ & $3.7+/-0.6$ & $4.5+/-0.7$ \\
\hline $\begin{array}{c}\text { LSG } \\
(n=12)\end{array}$ & $1.7+/-0.3$ & $2.1+/-0.5$ & $3.3+/-0.6$ & $3.9+/-0.5$ \\
\hline
\end{tabular}

Figure 3 


\begin{tabular}{|c|c|c|c|}
\hline & $\begin{array}{l}\text { Total patients who } \\
\text { underwent bariatric } \\
\text { surgery }(n=30)\end{array}$ & $\begin{array}{l}\text { Patients who } \\
\text { underwent RYGB } \\
\qquad(\mathrm{n}=18)\end{array}$ & $\begin{array}{l}\text { Patients who } \\
\text { underwent LSG } \\
\qquad(\mathrm{n}=12)\end{array}$ \\
\hline Age (years) & $51(35-56)$ & $51(43-56)$ & $40(31-57)$ \\
\hline Male sex & $10(33.3 \%)$ & $7(38.9 \%)$ & $3(25 \%)$ \\
\hline Arterial hypertension & $8(26.7 \%)$ & $7(38.9 \%)$ & $1(8.3 \%)$ \\
\hline Type 2 diabetes & $5(16.7 \%)$ & $3(16.7 \%)$ & $2(16.7 \%)$ \\
\hline Dyslipidemia & $10(33.3 \%)$ & $7(38.9 \%)$ & $3(25 \%)$ \\
\hline Hyperuricemia & $4(13.3 \%)$ & $2(11.1 \%)$ & $2(16.7 \%)$ \\
\hline Sleep apnea & $12(40 \%)$ & $8(44.4 \%)$ & $4(33.3 \%)$ \\
\hline Yo-yo effect & $14(46.7 \%)$ & $10(55.6 \%)$ & $4(33.3 \%)$ \\
\hline Active smoking & $5(16.7 \%)$ & $3(16.7 \%)$ & $2(16.7 \%)$ \\
\hline Past smoking & $7(23.3 \%)$ & $5(27.8 \%)$ & $2(16.7 \%)$ \\
\hline Body mass index $\left(\mathrm{kg} / \mathrm{m}^{2}\right)$ & $42.7(39.7-44.5)$ & $42.2(39.7-44.5)$ & $43.5(39.2-45.7)$ \\
\hline Weight $(\mathrm{kg})$ & $111.7(104.4-123.1)$ & $111.7(105.5-123.1)$ & $111.6(94.9-129.5$ \\
\hline Fat mass $(\%)$ & $51.5(44.5-55.3)$ & $52(45-54.5)$ & $47(42.3-59.5)$ \\
\hline Systolic blood pressure $(\mathrm{mmHg})$ & $121(120-130)$ & $121(120-132.5)$ & $122(112.5-128)$ \\
\hline Diastolic blood pressure (mmHg) & $74(70-80.3)$ & $80(68-82.5)$ & $71(70-78.5)$ \\
\hline Abdominal perimeter $(\mathrm{cm})$ & $130(125.5-137.3)$ & $131(122.3-138.5)$ & $129.5(126.3-136)$ \\
\hline
\end{tabular}




\begin{tabular}{|c|c|c|c|}
\hline Fasting glycemia (mmol/L) & $5.2(4.7-5.8)$ & $5.3(4.8-6.9)$ & $5.2(4.6-5.6)$ \\
\hline Insulinemia (mU/L) & $21.5(16.8-33.8)$ & $29(17.5-38)$ & $20.5(16.3-28)$ \\
\hline C-peptide (pmol/L) & $876.5(692.8-1083.3)$ & $942(759.3-1219.8))$ & $794(577.3-970)$ \\
\hline $\mathrm{HbA1c}$ & $5.6(5.3-6.1)$ & $5.6(5.3-6.5)$ & $5.6(5.3-6.1)$ \\
\hline HOMA- IR & $6.3(3.9-7.5)$ & $6.4(4-9.9)$ & $5.1(3.7-6.5)$ \\
\hline Total cholesterol (g/L) & $2.0(1.9-2.3)$ & $2.1(1.8-2.7)$ & $2.0(1.9-2.2)$ \\
\hline Triglyceride (g/L) & $1.4(0.9-1.9)$ & $1.5(1.1-1.9)$ & $1.1(0.8-2.2)$ \\
\hline HDL-cholesterol (g/L) & $0.5(0.4-0.6)$ & $0.5(0.4-0.6)$ & $0.5(0.4-0.5)$ \\
\hline LDL-cholesterol (g/L) & $1.3(1.0-1.6)$ & $1.3(0.9-1.8)$ & $1.3(1.1-1.3)$ \\
\hline Leptin (ng/mL) & $60.9(40.7-81)$ & $59.5(35-81)$ & $61.5(41.6-88.2)$ \\
\hline AST (U/L) & $24.5(20.8-33.8)$ & $25.5(20.8-36.8)$ & $22.0(20.3-28.8)$ \\
\hline ALT (U/L) & $35(23-51.3)$ & $39.5(23.8-52.8)$ & $26.5(21.5-43.3)$ \\
\hline GGT (U/L) & $33(17.8-61.8)$ & $39.5(21.8-54.3)$ & $28(12-63.3)$ \\
\hline Alkaline phosphatase (U/L) & $68(57.8-81.3)$ & $60(56-75.3)$ & $79.5(67-90.5)$ \\
\hline Total bilirubin $(\mu \mathrm{mol} / \mathrm{L})$ & $7.5(5.8-9.3)$ & $7(5-10)$ & $8(6-8)$ \\
\hline Conjugated bilirubin $(\mu \mathrm{mol} / \mathrm{L})$ & $3(2-4)$ & $3(2-4)$ & $2.5(2-3)$ \\
\hline
\end{tabular}

Table 1: Baseline characteristics of obese patients who underwent bariatric surgery. Values are expressed as median (interquartile range) or $\mathrm{n}(\%) . \%$ are expressed relative to each group. 


\begin{tabular}{|c|c|c|}
\hline $\begin{array}{c}\text { Variations of metabolic } \\
\text { parameters }\end{array}$ & Spearman coefficient correlation ( $r$ ) & P value \\
\hline BMI & 0.22 & 0.24 \\
\hline Fat mass & 0.22 & 0.25 \\
\hline Abdominal circumference & 0.37 & 0.04 \\
\hline Fasting glycemia & -0.16 & 0.40 \\
\hline $\mathrm{HbA1c}$ & -0.04 & 0.84 \\
\hline Insulinemia & -0.10 & 0.60 \\
\hline C-peptide & 0.06 & 0.75 \\
\hline HOMA-IR & -0.13 & 0.50 \\
\hline Triglyceride & -0.09 & 0.62 \\
\hline HDL-cholesterol & 0.10 & 0.59 \\
\hline
\end{tabular}

Table 2: Correlations between fasting glicentin variations after bariatric surgery and variations of the other metabolic parameters. Variations of glicentin and metabolic parameters were expressed as the ratio between 12 month-post-operative values reported to pre-operative values. 
Supplemental data

a

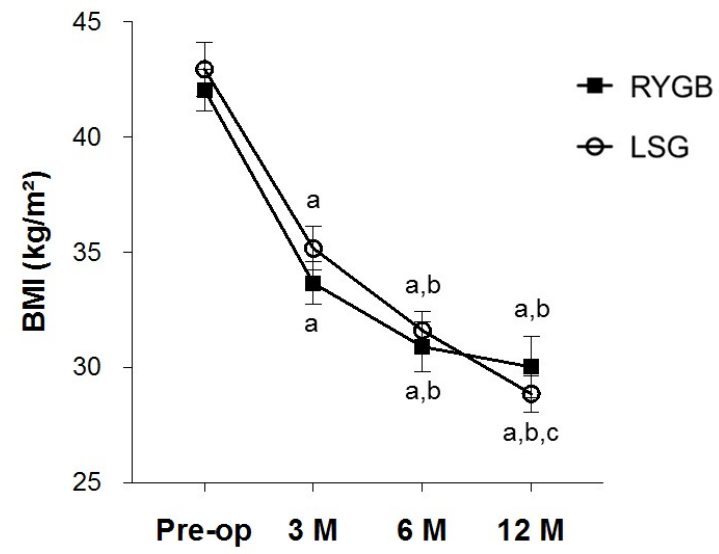

c

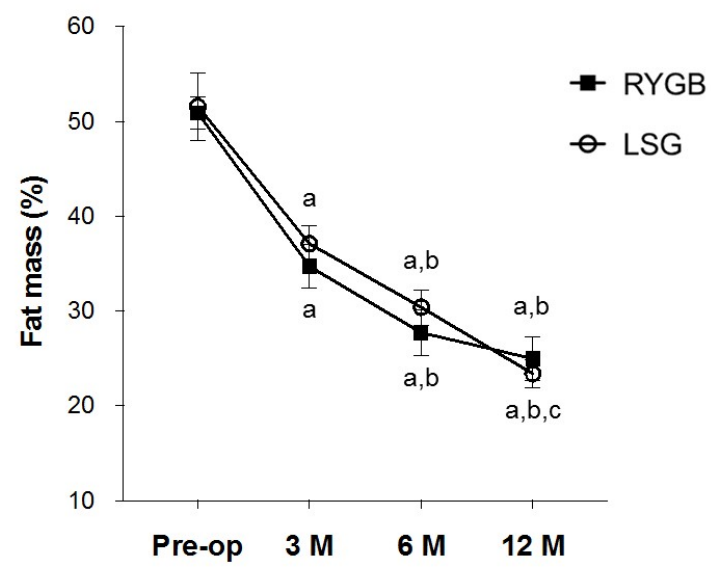

b

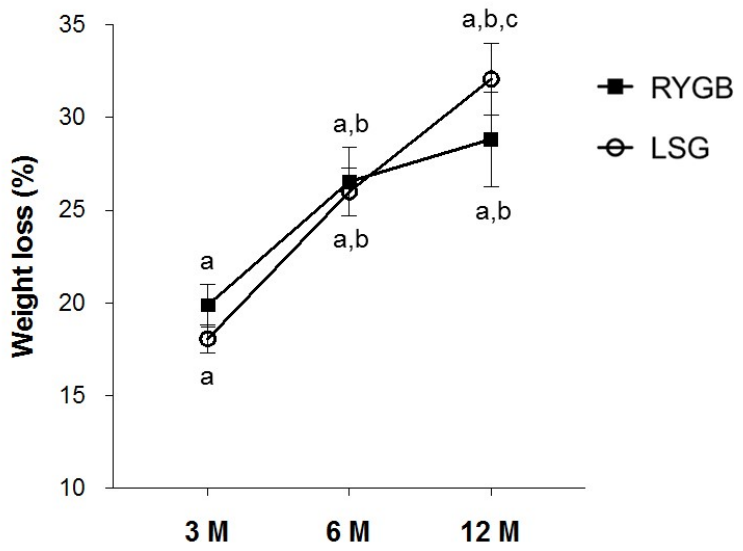

d

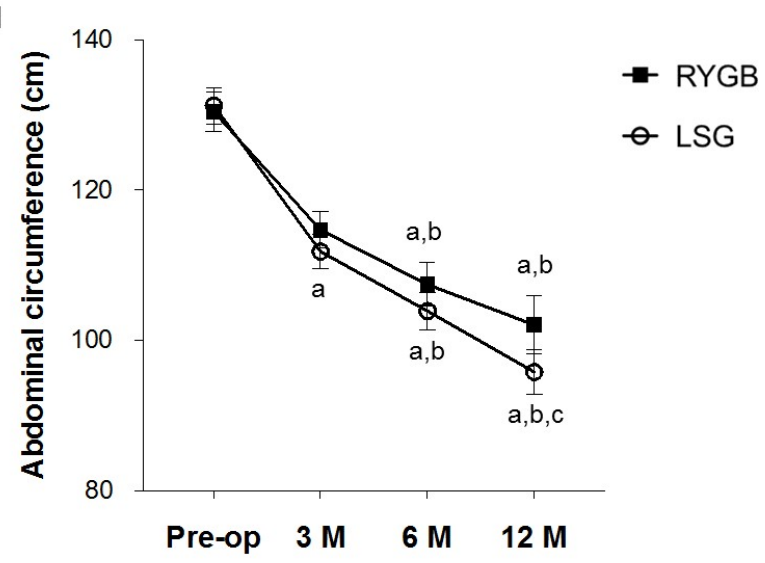

Figure S1 
a

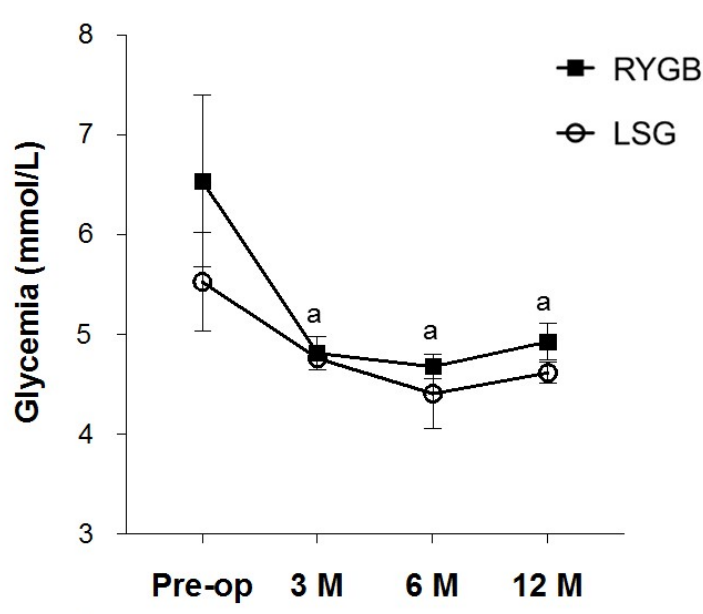

c

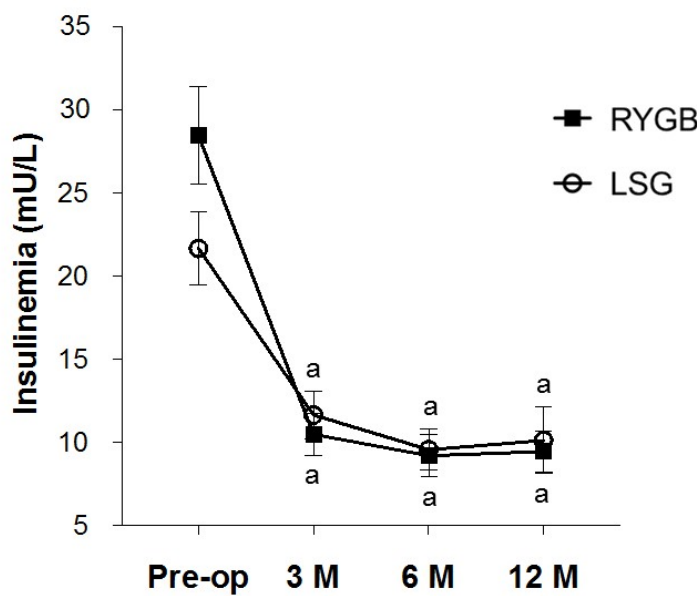

e

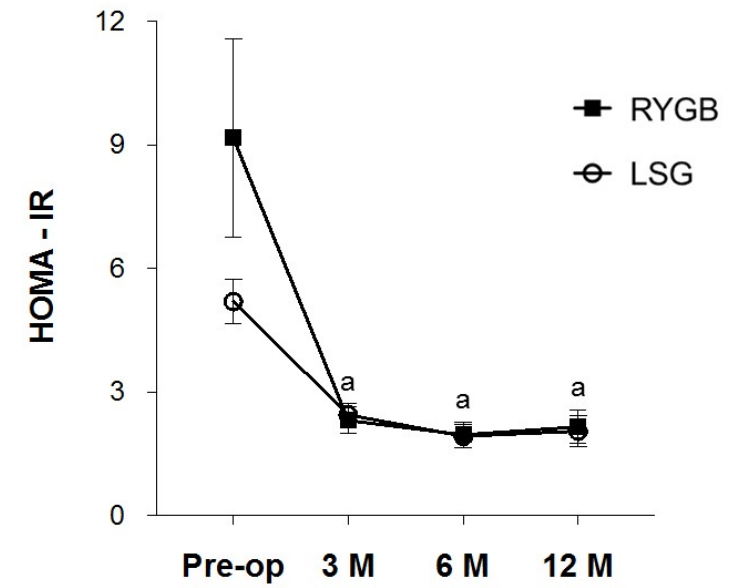

b

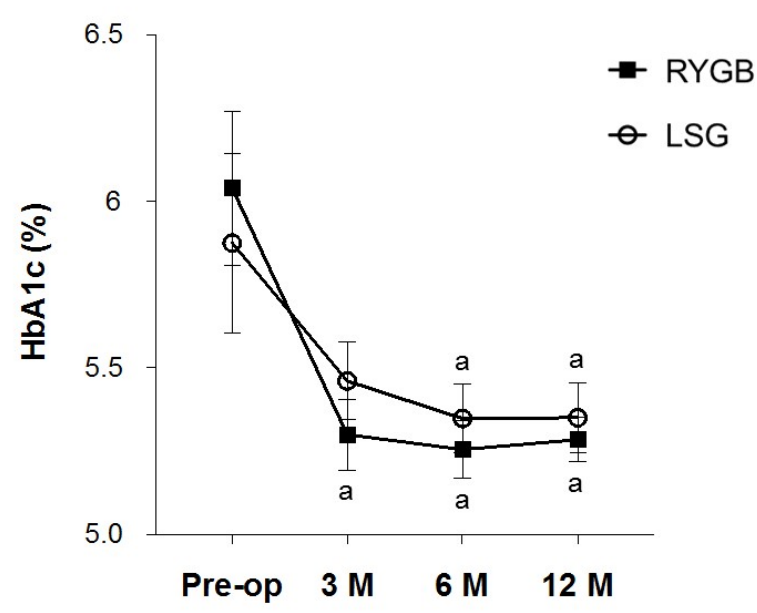

d

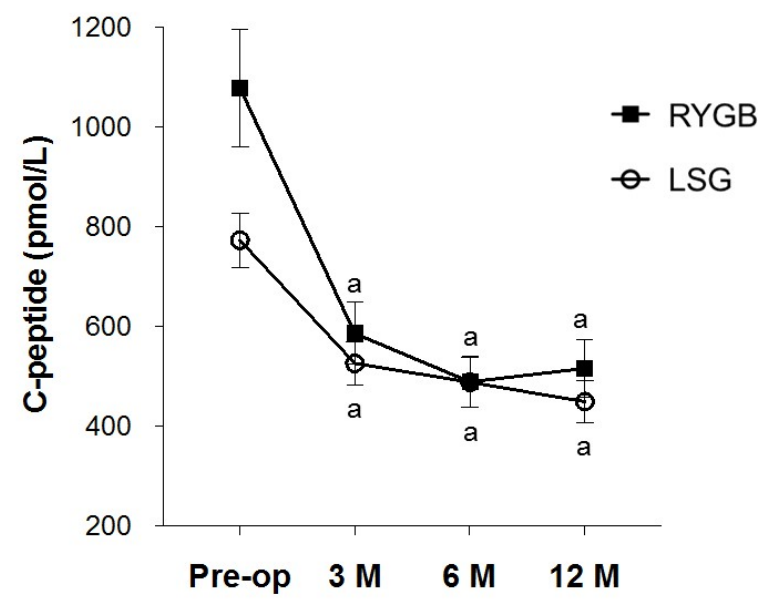

Figure S2 
a

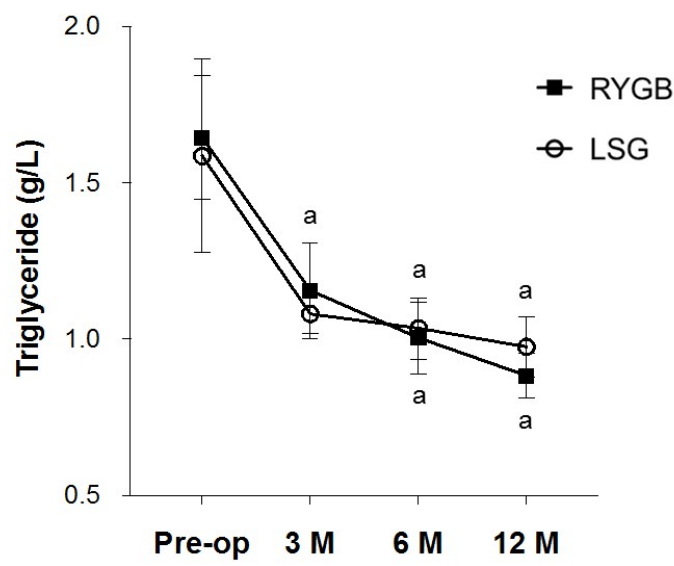

C

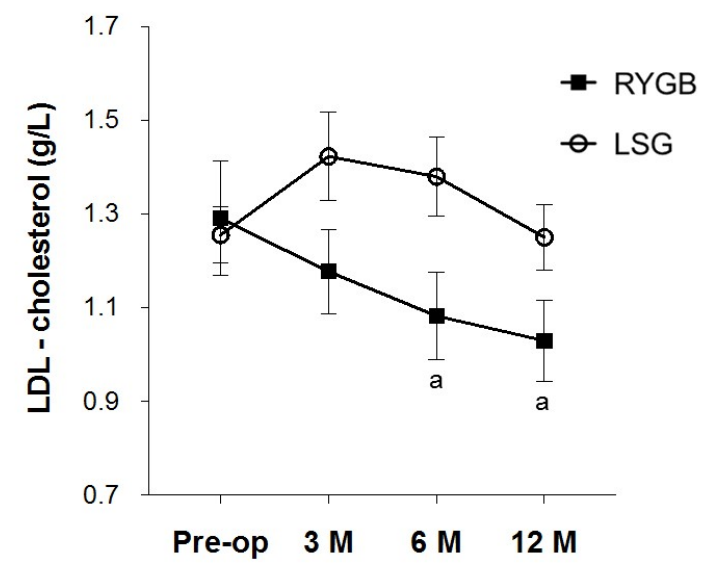

b

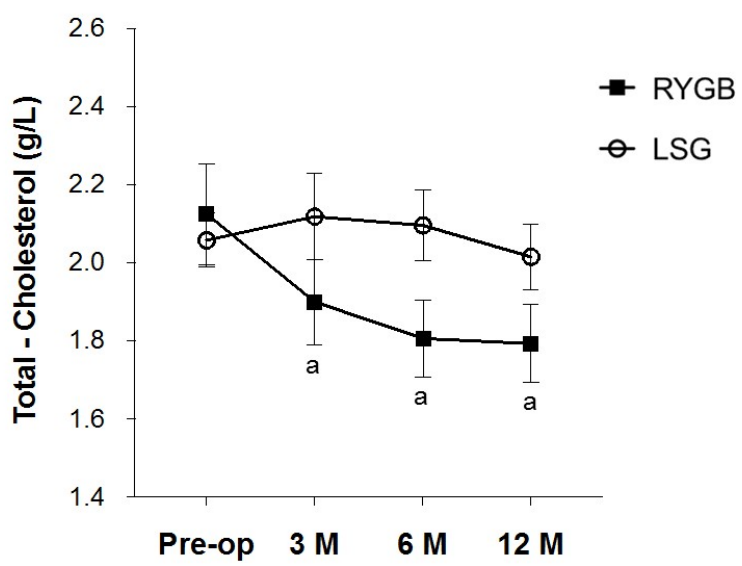

d

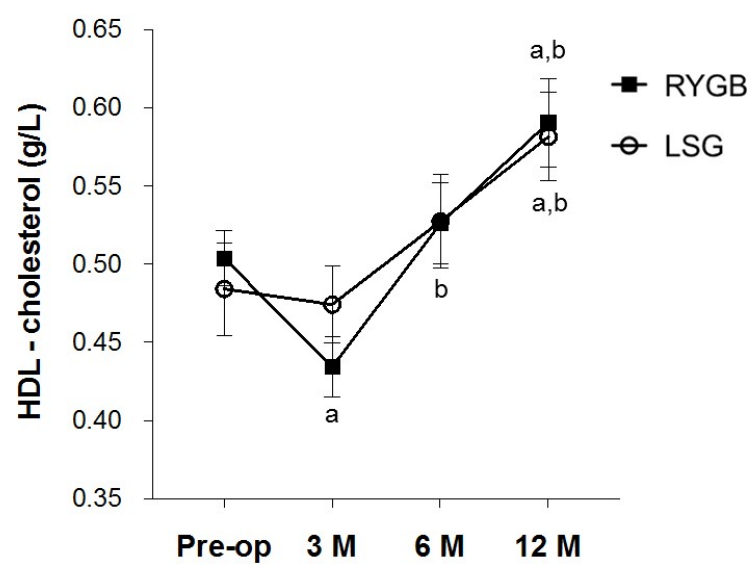

Figure S3 